\title{
Impact of a Simulation-Based Training on Self Assessed Nursing Performance in Anaphylactic Shock Management: A Pre-Post Educational Intervention Study
}

Qi Ren

Zhejiang Hospital

Fang Chen ( 2589087027@qq.com )

Zhejiang Hospital

Huijuan Zhang

Zhejiang Hospital

Juanhua Tu

Zhejiang Hospital

Xiaowei Xu

Zhejiang Hospital

Caixia Liu

Zhejiang Hospital

\section{Research Article}

Keywords: Graduated nurses, Anaphylactic shock, Rescue, Simulation-based training

Posted Date: September 30th, 2021

DOl: https://doi.org/10.21203/rs.3.rs-936444/v1

License: (c) (i) This work is licensed under a Creative Commons Attribution 4.0 International License.

Read Full License 


\section{Abstract}

Background: The New Nurses who lack the ability to recognize and manage anaphylactic shock can endanger the patients. In this study, we explored the effect of a simulated scenario designed to improve nurses' understanding of their roles and responsibilities during the rescue of a patient with anaphylactic shock.

Methods: The program of a simulation-based training was designed to teach learners to recognize the signs and symptoms of anaphylactic shock, place the patient in the correct position, stop ongoing intravenous infusion of the antibiotic which trigger the anaphylactic shock, restart an intravenous infusion on a new infusion apparatus, give $100 \%$ oxygen via a nasal cannula or mask, preserve airway patency, call the rapid response team (RRT), and correctly administer the medications prescribed by the clinician. Instructors evaluated each learner's skills and behaviors by using a clinical competency questionnaire. All learners then completed the Chinese version of the Simulation Design Scale(SDS) and participated in semi-structured interviews with their instructors after the training.

Results: All learners showed significant improvements in the 6 competencies assessed by the clinical competency questionnaire after the simulation-based training (all $P<0.001$ ). Scores on the SDS revealed that the learners were highly satisfied with all aspects of the simulation-based training (the 20 satisfaction rates were all above $90.00 \%$ ). During the semi-structured interviews, new graduated nurses reported that simulation-based training in the management of anaphylactic shock was extremely important and would guide them in clinical practice.

Conclusions: The simulation-based training in anaphylactic shock is a potentially viable and effective method to teach new registered nurses to manage clinical incidents.

\section{Background}

Anaphylactic shock is a severe potentially fatal hypersensitivity reaction that requires immediate treatment [1]. It can be triggered by a variety of antigens including venom of some insects and reptiles, antibiotics, nonsteroidal anti-inflammatory drugs (NSAIDs), glucocorticoids, opiates, vaccines, foods, and physical factors such as cold and exercise [2-4]. New qualified inexperienced nurses may feel overwhelmed and fail to rescue patients who experiencing anaphylactic shock. Nurses who are unable to recognize the signs and symptoms of anaphylactic shock and lack of the ability to manage it that can endanger the patients. Therefore, there remains an unmet need for education interventions that aim to increase knowledge of anaphylactic shock in graduated nurses.

Traditional classroom-based teaching is insufficient to train nurses to provide safe and competent professional care for patients with anaphylactic shock [5]. Evidence suggests that simulation-based training can improve nurses' clinical knowledge and performance in recognizing and responding to clinical emergencies [6-11]. The study based on simulated scenarios or cases can replicate real clinical situations, prompting learners to solve clinical problems and make key decisions. In China, opportunities 
to participate in simulation-based training in medical schools are limited. As a result, most graduated nurses have little experience with time-critical situations, such as anaphylactic shock. This study described a simulated scenario of anaphylactic shock that gave some graduated nurses an opportunity to assess and manage critically ill patients with anaphylactic shock in a safe environment without putting the patient at risk. Findings should inform the development of teaching approaches that facilitate the early identification and management of anaphylactic shock and improve the clinical competency of new registered nurses.

\section{Methods}

\section{Program implementation}

All learners were new registered nurses contracted to work for one year in a general hospital of Zhejiang province in China. Nurses who were unable to participate in the training because of illness or maternity leave were excluded from this research. All 104 new registered nurses contracted to work at the hospital were fully aware of the study procedures and volunteered to participate.

All Instructors were nurses with 10 years of clinical nursing experience and 3 years of clinical preceptor experience. They had all been awarded simulation-based teaching certificates after completing the Improving Simulation Instructional Methods (ISIM) program developed at the University of Pittsburgh.

The program of the simulation-based training was developed according the Allergic Shock and Anaphylactic Shock Rescue Protocol. The content was designed to teach learners to recognize the signs and symptoms of anaphylactic shock, place the patient in the correct position, stop ongoing intravenous infusion of the antibiotic which trigger the anaphylactic shock, restart an intravenous infusion on a new infusion apparatus, give $100 \%$ oxygen via a nasal cannula or mask, preserve airway patency, call the rapid response team (RRT), and correctly administer the medications prescribed by the clinician (Table 1).

Table 1

Implementation of the program of simulation-based training

\begin{tabular}{|llc|}
\hline Phase & Content & Time \\
\hline 1. Introduce the simulation & Training questions & $1 \mathrm{~min}$ \\
\hline 2. Recognize allergic shock & Nursing assessment & $2 \mathrm{mins}$ \\
\hline 3. Position training & Correct positioning of a patient with allergic shock & $1 \mathrm{~min}$ \\
\hline 4. Call the RRT & Familiar with the RRT's number and can call it correctly & $1 \mathrm{~min}$ \\
\hline 5. Oxygen & Giving oxygen about 4-6 L/min & 2 mins \\
\hline 6. Medications & Steroids given per MD's order & 2 mins \\
\hline 7. Summaries & Training feedback & $10 \mathrm{mins}$ \\
\hline
\end{tabular}


All learners were randomized divided into 10 groups, each with one instructor. Before the training, they were introduced to the principles of managing anaphylactic shock by the pre-briefing in the classroom. Immediately after the in-class session, the instructors conducted simulation-based training with each learner, one on one. The training lasted 20 minutes.

The training was conducted in every one of the ten rooms which were all equipped with a bed, a bedside table, a chair, a standardized patient (SP) and clinical supplies including a nasal cannula, an oxygen tubing, an mask, a bag valve, a treatment cart, an intravenous pump, a peripheral IV set, and some injection needles. Medications such as dexamethasone, normal saline and Lactate Ringer's solution would also be found in them.

The simulation room resembled a room of a cardiac ICU. The instructor introduced the scenario to the learners: "The patient is 68 years old, male, and have a history of hypertension and coronary heart disease. He was admitted to hospital due to dizziness and chest pain. This is a safe environment that will not cause harm to the patient and you can safely perform your duties. You are his head nurse, and when the patient is not feeling well, he will ring for you." 10 minutes after intravenous antibiotic, the patient developed chest pain, sweating, and purple lips. His vital signs: heart rate was 95 beats per minute, blood pressure was $60 / 40 \mathrm{mmHg}$, body temperature was $36^{\circ} \mathrm{C}$. The patient was flushed, delirious, and gasping for breath. There was no swelling of the lower extremities.

The learners were assured that the simulation provided a safe environment in which they could increase their clinical experience. The goal for learners was to stop the intravenous infusion of the antibiotic, restart an intravenous infusion of normal saline, give the patient oxygen, maintain the airway, call the RRT, and administer steroids as prescribed by the clinician. The instructors observed whether the learners followed the rescue procedure for anaphylactic shock correctly.

After the training, the learners were provided with the opportunity to review their simulation experience through instructor-guided debriefing following the "Gather, Analysis, Summarize format (GAS)". The debriefing began with a brief description of the anaphylactic shock and its rescue protocol to gather information and ensure all learners had a shared mental model of the events that had occurred. The instructors asked the learners: "Can you recall what you have done, what do you think?" Next, the anaphylactic shock rescue protocol was analyzed through using a "Plus-delta" method, in which the learners reflected on what went well (plus) and what could have been improved (delta). Instructor-guided questions included "I noticed that you opened the air way, why?" and "Besides calling bed side doctors, who else do you need to notify, and how?" The learners responded with "I noticed that the patient had a difficult breathe and worried about a possible swollen pharynx" and "I should call the RRT immediately". The debriefing concluded with learners' summary of the important points had been learned and discussing about that how they will change their performance in subsequent anaphylactic shock scenarios.

\section{Program assessment}




\section{Assessment questionnaire of the rescue for anaphylactic shock}

Before and after the simulation-based training, a clinical competency questionnaire was used by the instructors to evaluate each learner's skills and behaviors. The questionnaire addressed six competencies (assessment skills and ability to place the patient in a correct position, call the RRT, maintain an airway, administer oxygen and other therapies), each of which was scored "Yes" or "No" by the instructor.

\section{Learner feedback}

The Chinese version of the Jeffries Simulation Design Scale (SDS)

The Chinese version of the Jeffries Simulation Design Scale (SDS) was translated by Zhu FF. et al [12]. The scale has a good reliability (the total Cronbach's $\square$ was 0.948 ) and validity (the content validity index was above 0.830). The scale was designed to evaluate a simulation-based training, optimize teaching programs, and improve teaching quality. After the simulation-based training, the SDS was provided to the learners to obtain quantitative data about the simulation. The instrument includes 20 items that assess the features of the simulation-based training, from 5 dimensions: 1) objectives/information, 2) support, 3) problem solving, 4) feedback, and 5) fidelity. Those items were rated on a 5-point Likert scale ranging from "disagree (0)" to "strongly agree (5)".

\section{Semi-structured interviews}

After the simulation-based training, the instructors conducted the semi-structured interviews with the learners to elicit feedback on the program of the simulation-based training. Questions included: What have you learned from this training? What do you think about this training? After reviewing what you have done during this training, which part did you do well and which part you can do better in the future? According to what you have learned from this training, will you change your care during your clinical practice and how can you apply what you have learned to your daily clinical practice?

\section{Statistical analyses}

Epidata 3.1 software was used to establish a database, and double-entry verification was conducted to ensure the accuracy of data entry. SPSS 22.0 software was used for the statistical analysis.

Measurement data was reported as $(x \pm s d)$ or $\left[M\left(P_{25}-P_{75}\right)\right]$. Count data was reported as percentages. McNemar's test was used to compare data collected before and after the training. $P<0.05$ was considered statistically significant.

\section{Results}

The learners included 104 new graduated nurses (100 females and 4 males) with a mean age of 21.91 \pm 2.05 years (range 21-28 years). 13 learners had an Associate's degree in nursing, 90 learners had a Bachelor's degree in nursing, and 1 learner had a Master's degree in nursing. 
Assessment questionnaire of rescue for anaphylactic shock

All learners showed significant improvements in the accuracy of 6 competencies (assessment skills and ability to place the patient in a correct position, calling the RRT, maintaining an airway, administering oxygen and other therapies) assessed on the Assessment questionnaire of rescue for anaphylactic shock after the training (All $P<0.001$. Table 2). 
Table 2

The outcomes of the Assessment questionnaire of rescue for anaphylactic shock before and after the training $(n=104)$

\begin{tabular}{|c|c|c|c|c|c|}
\hline \multirow{3}{*}{$\begin{array}{l}\text { Items } \\
\text { Learners' assessment skills }\end{array}$} & \multicolumn{4}{|c|}{ Outcomes of the accuracy } & \multirow{6}{*}{$\begin{array}{l}P \text { value of McNemar's } \\
\text { test } \\
<0.001\end{array}$} \\
\hline & \multirow[t]{2}{*}{$\begin{array}{l}\text { Before the } \\
\text { training }\end{array}$} & \multicolumn{2}{|c|}{$\begin{array}{l}\text { After the } \\
\text { training }\end{array}$} & \multirow[t]{2}{*}{ Total } & \\
\hline & & Yes & No & & \\
\hline & Yes & 68 & 0 & 68 & \\
\hline & No & 34 & 2 & 36 & \\
\hline & Total & 102 & 2 & 104 & \\
\hline \multirow[t]{5}{*}{ Calling the RRT } & $\begin{array}{l}\text { Before the } \\
\text { training }\end{array}$ & \multicolumn{2}{|c|}{$\begin{array}{l}\text { After the } \\
\text { training }\end{array}$} & Total & $<0.001$ \\
\hline & & Yes & No & & \\
\hline & Yes & 75 & 0 & 75 & \\
\hline & No & 28 & 1 & 29 & \\
\hline & Total & 103 & 1 & 104 & \\
\hline \multirow[t]{5}{*}{$\begin{array}{l}\text { Maintaining patents' } \\
\text { airway }\end{array}$} & $\begin{array}{l}\text { Before the } \\
\text { training }\end{array}$ & \multicolumn{2}{|c|}{$\begin{array}{l}\text { After the } \\
\text { training }\end{array}$} & Total & $<0.001$ \\
\hline & & Yes & No & & \\
\hline & Yes & 41 & 0 & 41 & \\
\hline & No & 61 & 2 & 63 & \\
\hline & Total & 102 & 2 & 104 & \\
\hline \multirow[t]{5}{*}{ Shock position } & $\begin{array}{l}\text { Before the } \\
\text { training }\end{array}$ & \multicolumn{2}{|c|}{$\begin{array}{l}\text { After the } \\
\text { training }\end{array}$} & Total & $<0.001$ \\
\hline & & Yes & No & & \\
\hline & Yes & 58 & 1 & 59 & \\
\hline & No & 41 & 4 & 45 & \\
\hline & Total & 99 & 5 & 104 & \\
\hline \multirow[t]{2}{*}{ Administration of oxygen } & $\begin{array}{l}\text { Before the } \\
\text { training }\end{array}$ & \multicolumn{2}{|c|}{$\begin{array}{l}\text { After the } \\
\text { training }\end{array}$} & Total & $<0.001$ \\
\hline & & Yes & No & & \\
\hline
\end{tabular}




\begin{tabular}{|c|c|c|c|c|c|}
\hline \multirow[t]{4}{*}{ Items } & \multicolumn{4}{|c|}{ Outcomes of the accuracy } & \multirow{4}{*}{$\begin{array}{l}P \text { value of McNemar's } \\
\text { test }\end{array}$} \\
\hline & Yes & 31 & 3 & 34 & \\
\hline & No & 55 & 15 & 70 & \\
\hline & Total & 86 & 18 & 104 & \\
\hline \multirow[t]{5}{*}{$\begin{array}{l}\text { Administration of } \\
\text { medications }\end{array}$} & $\begin{array}{l}\text { Before the } \\
\text { training }\end{array}$ & \multicolumn{2}{|c|}{$\begin{array}{l}\text { After the } \\
\text { training }\end{array}$} & Total & $<0.001$ \\
\hline & & Yes & No & & \\
\hline & Yes & 40 & 0 & 40 & \\
\hline & No & 60 & 4 & 64 & \\
\hline & Total & 100 & 4 & 104 & \\
\hline
\end{tabular}

\section{Learners' feedback}

After the training, all learners completed the Chinese version of SDS in a timely manner and reflected on the training during the semi-structured interviews with their instructors.

The outcomes of the SDS revealed that the learners were highly satisfied with all aspects of the training (Satisfaction rate is equal to the number of learners who chose 4 and 5 divided by the total number of learners. Table 3). 
Table 3

Learners' feedback on simulation design characteristics: Simulation Design Scale $(n=104)$

Item

$\begin{array}{llllll}1 & 2 & 3 & 4 & 5 & \begin{array}{l}\text { Satisfaction } \\ \text { rate }\end{array}\end{array}$

I had adequate pre-training preparation and I was

$\begin{array}{lllll}0 & 0 & 2 & 7 & 95\end{array}$

$98.08 \%$ encouraged to participate.

I was provided clear and definite teaching goals.

$\begin{array}{llllll}0 & 0 & 1 & 5 & 98 & 99.04 \%\end{array}$

During the simulation teaching, I was provided enough

clinical information to facilitate me to solve the clinical

$\begin{array}{llllll}0 & 0 & 3 & 4 & 97 & 97.12 \%\end{array}$

problems.

I was provided enough clinical information during $\quad \begin{array}{lllllll}0 & 1 & 2 & 2 & 99 & 97.12 \%\end{array}$ simulation training.

The examples during teaching training have prepared me to $\quad \begin{array}{llllll}0 & 2 & 4 & 2 & 96 & 94.23 \%\end{array}$ understand the simulation training.

I was provided adequate support and help during the $\quad \begin{array}{lllllll}0 & 0 & 2 & 1 & 101 & 98.08 \%\end{array}$ simulation training.

Teachers can identify my needs when I need it.

$\begin{array}{llllll}0 & 0 & 2 & 2 & 100 & 98.08 \%\end{array}$

Teachers have been very helpful during the simulation training.

I had all the support during my entire training.

$\begin{array}{llllll}0 & 0 & 2 & 4 & 98 & 98.08 \%\end{array}$

My problem-solving skills have been improved after this simulation training.

I was encouraged to search all the possibilities of solving $\quad \begin{array}{llllll}0 & 1 & 3 & 5 & 95 & 96.15 \%\end{array}$ problems during this simulation training.

This simulation training was designed according to my knowledge and clinical skills.

Simulation teaching have provided the opportunities for me $\quad \begin{array}{llllll}0 & 0 & 3 & 4 & 97 & 97.12 \%\end{array}$ to improve my clinical assessment and nursing skills.

Simulation training have provided me constructive feedback $\begin{array}{lllllll}0 & 0 & 2 & 4 & 98 & 98.08 \%\end{array}$ upon making clinical nursing goals.

Structured and well-organized feedback.

$\begin{array}{llllll}0 & 0 & 0 & 5 & 99 & 100 \%\end{array}$

I was giving feedback promptly during simulation training.

$\begin{array}{llllll}0 & 0 & 2 & 5 & 97 & 98.08 \%\end{array}$

I was allowed to analyze my own clinical performance during feedback session.

I was given feedback from training preceptors after simulation teaching finished.

$\begin{array}{llllll}0 & 0 & 1 & 3 & 100 & 99.04 \%\end{array}$

$\begin{array}{llllll}0 & 0 & 0 & 6 & 98 & 100 \%\end{array}$

Items were rated on a five-point scale ranging from disagree (1) to strongly agree (5). 


\begin{tabular}{|l|ccccccc|}
\hline Item & $\mathbf{1}$ & $\mathbf{2}$ & $\mathbf{3}$ & $\mathbf{4}$ & $\mathbf{5}$ & $\begin{array}{l}\text { Satisfaction } \\
\text { rate }\end{array}$ \\
\hline $\begin{array}{l}\text { This simulation training was imitated by real clinical } \\
\text { environment. }\end{array}$ & 0 & 1 & 3 & 2 & 98 & $96.15 \%$ \\
\hline $\begin{array}{l}\text { The simulation training is real to me. } \\
\text { Items were rated on a five-point scale ranging from disagree (1) to strongly agree (5). }\end{array}$ & \\
\hline
\end{tabular}

The learners' reflection during semi-structured interviews demonstrated that they all believed that the simulation-based training in the management of anaphylactic shock is extremely important for new graduated nurses, and 103 (99.04\%) learners reported that the simulation-based training would guide them in clinical practice. All learners commented that instructors taught them how to safely handle emergency situations and resuscitate patients. 98 (94.23\%) learners felt more knowledgeable about activating RRT calls. 96 (92.30\%) learners reported improvements in their clinical judgment and emergency response. 80 (76.92\%) learners recognized that there was still room for improvement in some areas such as opening the airway and administration of the correct medications. When comparing simulation-based training to in-class teaching, 78 (74.29\%) learners reported that the simulation-based training improved their clinical judgment and emergency response successfully, and they hoped to participate in more simulation-based trainings.

\section{Discussion}

When faced with a critical incident, such as a patient in anaphylactic shock, nurses must make quick decisions despite in the complex, dynamic circumstances [13]. They must have situational awareness and automation so that they can perceive important information, comprehend present events, project future events, and avoid patients' harm caused by some preventable errors [14]. The present study describes a simulated scenario of anaphylactic shock. In this scenario, new graduated nurses were allowed to evaluate and manage a critically ill patient presenting with anaphylaxis in a safe environment that did not put a patient at risk. Following the simulation, the learners reported an improved understanding of their roles and responsibilities during the rescue of a patient with anaphylactic shock. Consistent with these findings, healthcare providers in a pediatric emergency unit provided a simulationbased training in the management of anaphylaxis by using a high-fidelity patient simulator mannequin and the training was better able to use epinephrine and refer patients to the allergy unit for evaluation [15], while nurses participating in clinical simulations of anaphylaxis in a hospital setting reported enhanced confidence in patient assessment skills, recognition of a problem, and accurate communication of findings to others on the healthcare team [16].

In healthcare, simulation-based training provides learner-centered educational opportunities that are designed to demonstrate procedures in an environment that mimics the reality of a clinical setting. Simulation allows an educator to control the learning environment, give immediate feedback, and promote learners' decision making and critical thinking skills. Simulation-based training provides a relatively safe teaching environment in which new learners can gain knowledge from their mistakes, 
develop the ability to complete tasks intuitively, and enhance their clinical competency $[17,18]$. Several studies have shown simulation-based training is superior to didactics and demonstration for teaching technical and nontechnical skills in healthcare providers [19-21].

In the present study, instructors' assessment of learners' clinical competency pre- and post-simulation showed significant improvements in learners' assessment skills and their ability to place the patient in the correct position, call the RRT, maintain an airway, and administer oxygen and other therapies. The learners developed the ability to recognize anaphylaxis, identify and remove the antibiotic, which was necessary to prevent clinical decompensation in the patient. They also realized the importance of promptly calling the RRT whose role is to intervene as early as possible in a deteriorating patient to avoid further, preventable critical events and so reduce the inhospital morbidity and mortality [22]. At present, multidisciplinary RRT have been successfully integrated into many hospitals resulting in improved resuscitation rates, increased morale and empowerment among nurses, redistribution of workload for nurses (reducing neglect of non-acutely ill patients during emergencies), and immediately access to expert help $[23,24]$. The learners became familiar with the resuscitation steps, including giving oxygen by face mask and aiming for an oxygen saturation above $92 \%$, and the treatment options for a patient with anaphylaxis, such as epinephrine, $\mathrm{H}_{1}$ antihistamines, $\mathrm{H}_{2}$ blockers for immunoglobulin $\mathrm{E}$ mediation, and administration of steroids.

The learners' evaluations of the simulation were very positive. Simulation-based training provided a realistic clinical experience that facilitated learners' professional development and increased their selfconfidence as members of a practicing healthcare team [25,26]. Most learners believed that the simulation mimicked the clinical environment and enhanced their understanding of early, rapid recognition and treatment of anaphylactic shock. $99.04 \%$ of the learners reported that the instructors had been helpful, while $97.12 \%$ of the learners reported that the instructors were supportive. This was especially important as some learners felt overwhelmed and helpless when faced with a patient who was having difficulty breathing, even during simulation. Previous studies have reported the efficacy of simulation-based training in nurses, indicating that simulation builds confidence, makes learners aware of the aspects of care that need to be improved through deliberate practice, increases nurses' motivation to learn and transfer their knowledge, and strengthens communication among team members [25, 2728].

This study had some limitations. Firstly, the sample size was not calculated prior to performing the study as a literature search revealed no similar research for a reference. However, all new registered nurses contracted to work at the hospital for one year participated in this study. Secondly, this study did not include a control group. The study aimed to improve the clinical competency of new registered nurses; therefore, a pretest posttest study design was adopted. Thirdly, learners' self-reports were used to evaluate the program of the training. Although their self-reports were easy to obtain, the approach is associated with response bias due to false reporting and variations in learners' capacity to interpret questions and their introspective ability. Fourthly, the Chinese version of the SDS has some limitations. The scale is not widely used, so its reliability may be questioned. A few items have factor loadings $<0.4$, 
which may have an impact on validity [12]. Lastly, the scenario was not validated as a reliable simulation of anaphylactic shock. However, the outcomes of this study suggest this simulation is applicable for training new registered nurses and warrants further development.

\section{Conclusions}

The simulation-based training which used an simulated scenario of anaphylaxis was feasible and effective as a method to teach new graduated nurses to manage clinical incidents of anaphylactic shock. It is able to built confidence in new graduated nurses, facilitating the provision of safe patient-centered care.

\section{Abbreviations}

\begin{tabular}{|ll|}
\hline Full name & Abbreviation \\
\hline Rapid response team & RRT \\
\hline Simulation Design Scale & SDS \\
\hline Nonsteroidal anti-inflammatory drugs & NSAIDs \\
\hline Improving Simulation Instructional Methods & ISIM \\
\hline Standardized patient & SP \\
\hline Intravenous injection & IV \\
\hline Intensive care unit & ICU \\
\hline Gather, Analysis, Summarize format & GAS \\
\hline
\end{tabular}

\section{Declarations}

\section{Ethical approval and consent to participate}

This study was approved by the ethics committee of Zhejiang Hospital. All procedures performed in studies involving human participants were in accordance with the ethical standards of the institutional and/or national research committee.

\section{Consent for publication}

This manuscript does not contain any individualized person's data.

\section{Availability of data and materials}

The data and materials of the present study are available from the corresponding author on reasonable request. 


\section{Competing interests}

The authors declare that they have no competing interests.

\section{Funding}

None.

\section{Authors' Contributions}

Qi Ren designed and performed most of the investigation, and wrote the manuscript; Caixia Liu, Juanhua $\mathrm{Tu}$, and Xiaowei Xu provided data analysis; Huijuan Zhang and Fang Chen contributed to interpretation of the data and analyses. All of the authors have read and approved the manuscript.

\section{Acknowledgements}

Not applicable.

\section{References}

1. Krčmová I, Novosad J. Anaphylactic symptoms and anaphylactic shock. Vnitr Lek. 2019; 65(2): 149156.

2. Reber LL, Hernandez JD, Galli SJ. The pathophysiology of anaphylaxis. J Allergy Clin Immunol. 2017; 140(2): 335-348.

3. Atanaskovic-Markovic M, Gomes E, Cernadas JR, et al. Diagnosis and management of drug-induced anaphylaxis in children: an EAACl position paper. Pediatr Allergy Immunol. 2019; 30(3): 269-276.

4. Christensen MJ, Eller E, Kjaer HF, et al. Exercise-induced anaphylaxis: causes, consequences, and management recommendations. Expert Rev Clin Immunol. 2019; 15(3): 265-273.

5. Zhang C, Mou S. Status and development of standardized training system for new graduate nurses in China. Chin J Nurs Educ. 2014; 8(11): 631-633.

6. Hegland PA, Aarlie H, Strømme H. Simulation-based training for nurses: Systematic review and metaanalysis. Nurse Educ Today. 2017; 54: 6-20.

7. Yunoki K, Sakai T. The role of simulation training in anesthesiology resident education. J Anesth. 2018; 32(3): 425-433.

8. McCoy CE, Rahman A, Rendon JC, et al. Randomized controlled trial of simulation vs. Standard training for teaching medical students high-quality cardiopulmonary resuscitation. West $\mathrm{J}$ Emerg Med. 2019; 20(1): 15-22.

9. Kato C, Kataoka Y. Simulation training program for midwives to manage postpartum hemorrhage: A randomized controlled trial. Nurse Educ Today. 2017; 51: 88-95.

10. Kishiki T, Su B, Johnson B, et al. Simulation training results in improvement of the management of operating room fires-A single-blinded randomized controlled trial. Am J Surg. 2019; 218(2): 237-242. 
11. Meischke H, Painter IS, Stangenes SR, et al. Simulation training to improve 9-1-1 dispatcher identification of cardiac arrest: A randomized controlled trial. Resuscitation. 2017; 119: 21-26.

12. Zhu FF, Wu L, Zhang D. Reliability and validity of the Chinese version of simulation design scale. Chin Nurs Manag. 2017; 17(12): 1620-1623.

13. White $C$, Chuah J, Robb A, et al. Using a critical incident scenario with virtual humans to assess educational needs of nurses in a postanesthesia care unit. J Contin Educ Health Prof. 2015; 35(3): 158-165.

14. Aun MV, Kalil J, Giavina-Bianchi P. Adults and children with anaphylaxis in the emergency room: why it is not recognized? Curr Opin Allergy Clin Immunol. 2018; 18(5): 377-381.

15. Barni S, Mori F, Giovannini M, et al. In situ simulation in the management of anaphylaxis in a pediatric emergency department. Intern Emerg Med. 2019; 14(1): 127-132.

16. Mason VM, Lyons P. Use of simulation to practice multidisciplinary anaphylaxis management. Dimens Crit Care Nurs. 2013; 32(6): 280-285.

17. Silveira MS, Cogo ALP. The contributions of digital technologies in the teaching of nursing skills: an integrative review. Rev Gaucha Enferm. 2017; 38(2): e66204.

18. Fathi A, Hajizadeh M. Medication errors among nurses in teaching hospitals in the west of Iran: what we need to know about prevalence, types, and barriers to reporting. Epidemiol Health. 2017; 39: e2017022.

19. Semler MW, Keriwala RD, Clune JK, et al. A randomized trial comparing didactics, demonstration, and simulation for teaching teamwork to medical residents. Ann Am Thorac Soc. 2015; 12(4): 512-519.

20. Everson J, Gao A, Roder C, et al. Impact of simulation training on undergraduate clinical decisionmaking in emergencies: a non-blinded, single-centre, randomised pilot study. Cureus. 2020; 12(4): e7650.

21. Maldonado MJ, Agudelo SI, Suarez JD, et al. Educational strategy for the development of skills in exchange transfusion: a randomized clinical trial protocol. Trials. 2020; 21(1): 387.

22. Danesh $V$, Neff $D$, Jones $T L$, et al. Can proactive rapid response team rounding improve surveillance and reduce unplanned escalations in care? A controlled before and after study. Int J Nurs Stud. 2019; 91: 128-133.

23. Elliott R, Martyn L, Woodbridge S, et al. Development and pragmatic evaluation of a rapid response team. Crit Care Nurs Q. 2019; 42(3): 227-234.

24. Arabi YM, Al-Dorzi HM, Alamry A, et al. The impact of a multifaceted intervention including sepsis electronic alert system and sepsis response team on the outcomes of patients with sepsis and septic shock. Ann Intensive Care. 2017; 7(1): 57.

25. Alamrani MH, Alammar KA, Alqahtani SS, et al. Comparing the effects of simulation-based and traditional teaching methods on the critical thinking abilities and self-confidence of nursing students. J Nurs Res. 2018; 26(3): 152-157. 
26. Almeida RGDS, Mazzo A, Martins JCA, et al. Self-confidence in the care of critically ill patients: before and after a simulated intervention. Rev Bras Enferm. 2019; 72(6): 1618-1623.

27. Hustad J, Johannesen B, Fossum $M$, et al. Nursing students' transfer of learning outcomes from simulation-based training to clinical practice: a focus-group study. BMC Nurs. 2019; 18: 53.

28. Lindsey PL, Jenkins S. Nursing students' clinical judgment regarding rapid response: the influence of a clinical simulation education intervention. Nursing forum. 2013; 48(1): 61-70. 